\title{
Development and validation of a multi- domain multimorbidity resilience index for an older population: results from the baseline Canadian Longitudinal Study on Aging
}

Andrew Wister ${ }^{1 *}$, Scott Lear ${ }^{2}$, Nadine Schuurman ${ }^{3}$, Dawn MacKey ${ }^{4}$, Barbara Mitchell ${ }^{5}$, Theodore Cosco ${ }^{6,7}$ and lan Fyffe $e^{8}$

\begin{abstract}
Background: Multimorbidity is recognized as a major public health issue that increases with age and affects approximately two-thirds of older people in Canada, the US, Australia and many European countries. This study develops and tests a three domain (functional, social and psychological) multimorbidity resilience composite index based on a previously developed lifecourse model of multimorbidity resilience, incorporating measures of adversity and positive adaptation. The criterion validity of the measure is demonstrated by means of an analysis of key outcome variables drawn from the literature.

Methods: We used the baseline data from the Comprehensive Cohort of the Canadian Longitudinal Study on Aging. Associations of functional, social, psychological as well as total resilience with two health utilization and three illness context outcome variables were examined using logistic regression analyses, adjusted for age, gender, marital status, income, education, region, and number of chronic conditions.

Results: The sample included all 6771 Canadian adults aged 65 or older (mean age 73.0, 57\% women) who reported two or more of 27 possible chronic conditions. Total resilience was associated with: perceived health (OR $=1.68, \mathrm{Cl} 1.59-1.77)$; sleep quality $(\mathrm{OR}=1.34, \mathrm{Cl} 1.30-1.38)$; perceived pain $(\mathrm{OR}=0.80, \mathrm{Cl} 0.77-0.83)$; hospital overnight stays ( $\mathrm{OR}=0.87, \mathrm{Cl} 0.83-0.91)$; and emergency department visits ( $\mathrm{OR}=0.90, \mathrm{Cl} 0.87-0.94)$., after adjusting for socio-demographic factors, and number of chronic conditions. These associations were similar for the unadjusted models, as well as for the functional, social and psychological resilience sub-indices.

Conclusions: Combining components of adversity and positive adaptation within functional, social and psychological domains produces a measure of multimorbidity resilience that is associated with more positive health outcomes. Several implications of a composite multimorbidity resilience measure for clinical practice are identified. This measure can be replicated using measures found in other secondary health data sets. Future validation using longitudinal data is warranted.
\end{abstract}

Keywords: Multimorbidity resilience index, Multi-domain, Validation

\footnotetext{
* Correspondence: wister@sfu.ca

'Department of Gerontology, and Gerontology Research Centre, Simon

Fraser University, 2800-515 Hastings Street, Vancouver, BC V6B 5K3, Canada

Full list of author information is available at the end of the article
}

(c) The Author(s). 2018 Open Access This article is distributed under the terms of the Creative Commons Attribution 4.0 International License (http://creativecommons.org/licenses/by/4.0/), which permits unrestricted use, distribution, and reproduction in any medium, provided you give appropriate credit to the original author(s) and the source, provide a link to the Creative Commons license, and indicate if changes were made. The Creative Commons Public Domain Dedication waiver (http://creativecommons.org/publicdomain/zero/1.0/) applies to the data made available in this article, unless otherwise stated. 


\section{Background}

Recently, there has been a growing interest in model developments aimed at understanding how individuals respond to illness-related adversities and regain a sense of wellness in their lives, termed resilience - the ability and resources needed to adapt and navigate stress-inducing experiences [1-3]. Although a broader literature has existed on resilience for decades applied to a variety of topics and populations [1, 4-7], research has been accumulating on specific substantive areas [8]. One of these is the application of resilience to older persons with more than one concurrent chronic disease termed multimorbidity [8-12]. Multimorbidity has been shown to significantly increase with advanced age, and affects individuals adversely on many levels (i.e., physiological, psychological, social), making it appropriate for the application of resilience models. For instance, US clinical data show that $62 \%$ of persons aged 65-74, $75.7 \%$ aged $75-84$, and $81.5 \%$ aged $85+$ have 2 or more of 15 prevalent chronic illnesses [13]. Although variable depending on the type of data, population, and number of chronic illnesses, similar patterns have been observed in Canada and Australia [14]. Given that individuals with multiple chronic diseases are unlikely to completely overcome these adversities due to their permanence, and that a positive response may entail adaptation (coping) and/or only partly "bounce back" from their effects, we term this multimorbidity resilience.

Numerous measures of resilience have been developed and applied to different research questions and populations, but dominated by psychological measures that have been used to study mental health conditions and outcomes among children and among the general population $[5,7,11]$. Also, measures of resilience have been highly variable, depending on their theoretical and/or conceptual roots, methodological construction, and application, and typically are not adapted to an older population with unique multimorbidity illness contexts. This leaves significant research gaps, given that resilience measures are primarily psychological in nature (i.e., affective states), or qualitative, rather than covering measurable content domains based on underlying strengths and vulnerabilities associated with an aging and illness lens [5]. This paper operationalizes multimorbidity resilience as the combination of three domains: functional, social and psychological resilience, comprised of adversity and resilience components, and examines the criterion validity of these measures using key outcome measures of health care utilization and illness context among a vulnerable population of older individuals with multimorbidity.

There is likely no greater challenge to healthy aging and health care systems than the concurrent experience of multiple illnesses $[15,16]$. Multimorbidity compounds the deleterious effects of living with individual chronic conditions by a synergistic exacerbating effect on symptom burden [17], lowering quality of life indicators, such as self-rated health and well-being $[8,18]$, and increasing complexity and cost of treatment $[15,16]$. Yet, a gap in the literature is that we have a limited understanding of multimorbidity adaptation, self-care/coping, and healthy aging. The advancement of resilience concepts and the development of new measures and applications would assist in filling this void. This work is particularly relevant given the large and growing population of adults with multimorbidity who are aging during a period of escalating health care costs. For these reasons, there is a need to develop resilience measures that capture positive adaptation to illness contexts experienced among older populations.

Moreover, multimorbidity literature has predominantly focused on the pathogenic correlates, treatments, and disablement outcomes of multimorbidity, or of associated conditions or deficits, such as frailty $[1,6]$. However, some individuals may possess important factors such as social support, economic resources and psychosocial strengths that may enable them to live well with and adapt to multiple chronic conditions [9, 10, 16, 19]. Although applications of resilience have often defined it in terms of recovery, the National Academy of Sciences has included adaptation as a central component that includes system reorganization, responses to stress, and social learning that can affect psychological resilience [20]. Applied to multimorbidity resilience, adaptation and coping may be more relevant than recovery, particularly given the chronic nature of multimorbidity in older age. Thus, there remains a significant gap in research that explicates the complexity of resilience types, processes, and determinants specific to the occurrence of chronic illness and disability in old age.

\section{Resilience, multimorbidity and aging}

Resilience has been conceptualized in variable forms, including psychological, emotional, spiritual, physical/ functional, economic, cultural, and social or ecological resilience $[1-3,6]$. One popular measure with established psychometric properties is the Connor-Davidson Resilience Score (either the ten item or two item version) [4]. Similar to many resilience scales, such as the Brief Resilient Coping Scale [21], the Connor-Davidson Score measures the degree to which individuals perceive that they can overcome stress and adversity in life through a general set of questions [4]. However, this measure assumes that there is a singular concept of resilience [5] and it has not been specifically applied to multimorbidity among older people exposed to heightened health-related adversity. Therefore, this measure may be limited in this sub-field, since the items are not 
anchored in the experience of chronic illness or multimorbidity among older adults - a condition that is slow in progression, long in duration, and typically limits function, productivity and quality of life $[15,16]$.

A common challenge is that any resilience measure entails combining levels of adversity with levels of positive response or adaptation, since resilience may be present but not activated without the occurrence of challenges. Some approaches have developed typologies based on ad hoc definitions of adversity thresholds and positive or negative responses [5]. Statistical or datadriven approaches have tended to use cross-sectional data, although some have used repeated-measure analyses of longitudinal data to identify a continuum of resilience, based on change in levels of adversity and adaptation [5]. Yet, there is no agreed upon approach to measuring resilience in the literature. While access to longitudinal data is preferable, there is a need for measurement development and testing that can be utilized in a broader set of studies and for specific populations $[5,7,11]$.

Applications to multimorbidity among older adults requires consideration of several unique elements. The present research attempts to tap into resilience by first identifying a sub-population with exposure to adversity - multimorbid older adults; and second, by combining both adversity and adaptation (coping) factors into a multi-domain multimorbidity resilience index. A significant body of research has accumulated demonstrating the importance of health-related quality of life (HRQL) indicators of successful and healthy aging among persons with multimorbidity $[7,12]$. A previous comprehensive systematic review of 112 articles (published between 1995 and 2015) supplemented with an additional 14 articles published since that time was conducted for the purpose of developing the Lifecourse Model of Multimorbidity Resilience (LMMR). In this study, we draw on the LMMR to identify a set of primary domains and associated measures of adversity and adaptation, and combine these into a composite index specifically applied to aging-related adversity and adaptation. The composite index (including sub-domains) is validated using outcomes identified in the multimorbidity literature.

The LMMR model connects several sources of resources embedded in the individual, family, community contexts, which can be harnessed in order to overcome the disablement processes of illness, and allow for individual reintegration and homeostasis. Adaptation/ resilience, as well as vulnerability/adversity processes, are understood as dynamic over an individual's lifecourse. The LMMR provides an overarching framework and rationale for three resilience domains, each of which contains both adversity and adaptation (resilience) components [12]. 1) Functional resilience is deemed to be fundamental to aging well as it relates to the ability of a multimorbid individual to complete tasks of daily living, social roles, and remain physically active $[1,6]$. Functional disability is a key aspect of the disablement process that can increase vulnerability and limit one's ability to maintain daily activities of living, healthy living, as well as remaining engaged in community. 2) Social resilience can be understood as a multimorbid individual's maintenance of positive social interaction, including community participation, as well as protecting against feelings of loneliness and experiencing social isolation that can result in negative adaptation. According to the LMMR, the successful activation of social resilience entails harnessing available resources, especially social support networks $[9,11]$. An external activation of social resources may include support from a friend or family member, or the utilization of social capital derived from community participation. Social isolation, on the other hand, is expected to result in low levels of multimorbidity social resilience and integration [12]. 3) Psychological resilience pertains to the ability to mentally cope with stressors associated with multimorbidity. The degree to which individuals perceive stress in the face of multimorbidity, experience degrees of depression, and maintain psychological well-being represent aspects of this domain [11, 19]. This type of resilience draws from stress theory and the cognitive appraisal process [22], wherein stressfulness and challenges that are faced in old age due to episodic pain and disability can lead to the disruption of self-concept, health behaviours and health care decisions. Alternatively, feelings of well-being or satisfaction with life can result in internal activation of resources that can help individuals overcome adversity associated with chronic illness [19].

Multimorbidity resilience domains are assumed to be interrelated, fluid, and modifiable over the life course of individuals. Validation of a multimorbidity resilience index requires consideration of the unique characteristics of the individual domains as well as a composite index comprised of all content areas. Criterion validity is also required to examine correlations between the sub-indices, total index, and anticipated outcomes. Research indicates that a measure of multimorbidity resilience should be correlated with perceived health, as well as measures of health care utilization $[9,10,16,17]$.

\section{Methods}

\section{Design and sample}

This research utilizes a subset of the Comprehensive Cohort (see below) of the Baseline Wave of the Canadian Longitudinal Study on Aging (CLSA) dataset. Launched in 2010, this 20 year panel study of persons aged 45 to 85 has been funded primarily by the Canadian Institutes for Health Research (CIHR), Canada's federal granting agency for health research. Data were being collected at 
baseline including biological, clinical, psychosocial and societal information that influence disease, health, and well-being [23]. The CLSA participants were randomly selected and invited to participate from the population aged 45 to 85 (excluding those living in institutions, full-time military, persons living on federal First Nations reserves and in the three northern territories), resulting in a total sample of 51,338 . Inclusion criteria entailed being able to complete the interviews in English or French, live in the community, and be cognitively functional. The sampling method is the same as used by Statistics Canada for its Canadian Community Health Survey (CCHS) - Healthy Aging 2008/09 survey, and included participants from that study in its recruitment, supplemented with Provincial Health Registries, telephone sampling using random digit dialling, and the Quebec Longitudinal Study on Nutrition and Aging. The sample contains weights to adjust for sampling error and to produce a sample that is representative of the targeted Canadian population.

The CLSA is comprised of two cohorts of participants: 21241 Tracking Cohort participants and 30,097 Comprehensive Cohort participants, each of which can be used separately, since the Tracking Cohort collected only telephone-based data, whereas the Comprehensive Cohort included physiological data as well as a face-to-face interview [23].

The present research uses only the Comprehensive Cohort $(n=30,097)$, since several physiological measures are only available in this cohort [21]. Comprehensive participants were randomly selected within age/sex strata from within $25 \mathrm{~km}$ of dense population data sites, or within $50 \mathrm{~km}$ of data collection sites in areas with a lower population density. The 11 data collection sites for the CLSA are located in Victoria, BC; Vancouver, BC; Surrey, BC; Calgary, AB; Winnipeg, MB; Hamilton, ON; Ottawa, ON; Montreal, QC; Sherbrooke, QC; Halifax, NS; and St. John's, NFLD.

Given our interest in developing and testing a multimorbidity resilience index among older adults, our study included only persons aged 65 or over who reported having two or more chronic conditions $(n=6771)$. Sample weights were used to correct for sampling error by age, gender, and geographic location. The sample self-reported two or more of 27 possible chronic conditions including Alzheimer's disease, back problems, bowel incontinence, cancer, cataracts, diabetes, epilepsy, glaucoma, heart attack, heart disease, high blood pressure, irritable bowel syndrome, kidney disease, Parkinson's disease, peripheral vascular disease, lung disease, macular degeneration, multiple sclerosis, osteoarthritis, osteoporosis, migraine headaches, rheumatoid arthritis, stroke, thyroid problem, transient ischemic attack, ulcer, and urinary incontinence. The validity and reliability of all relevant measures in the CLSA questionnaires, as well as references, can be found on the Data Portal of the CLSA web site (www.clsa-elcv.ca).

The independent variable with the largest amount of missing data was total household income $(n=610$ or $9.0 \%$ ). This variable was recoded using multiple imputation based on age, gender, education, and region. The remaining independent and dependent variables had minimal (under 3\%) to no missing cases. Missing data for these variables were recoded to the respective means or modes. Analyses were replicated when removing missing data; therefore, only the analyses with all missing imputed data are presented.

\section{Measurement Multimorbidity resilience index}

A multimorbidity resilience index was created based on a composite (additive) index of three sub-indices representing functional, social, and psychological multimorbidity resilience domains. Each of these three sub-indices was, in turn, comprised of three index domain measures of adversity challenges and positive adaptation. Measures were coded into positive resilience, such as that higher scores reflect greater resilience.

\section{Functional resilience variables}

The three functional variables were the Older Americans Resources and Services

(OARS) Activities of Daily Living (ADL) Scale [24], the OARS Instrumental Activities of Daily Living (IADL) Scale [22], as well as the Summary Performance Score of functional ability scale [24]. The Summary Performance Score used in this study was calculated including a standing balance measure, a walk time measure, and a timed chair raise measure. Similar to this measurement construction, participants who completed these three tasks were assigned scores per task ranging from 1 to 4 , which corresponded to statistical quartiles. Participants who did not complete a task were assigned a 0 , with a range of 0 to 12 [25]. These lower extremity function tests directly measure physical challenge.

The OARS ADL Scale consisted of 7 items [24] of such tasks as eating and bathing. Each question was measured on a scale from 0 (completely unable) to 2 (completely able). Possible total scores range from 0 to 14, with higher scores indicating greater functional status. Similarly, the 7 item OARS IADL Scale also assesses functional ability [24]. Scores for the OARS IADL Scale questions also range from 0 to 2 and utilized the same coding scheme as above. These tasks are considered to be instrumental to daily living such as taking medicine and meal preparation, and reflect positive adaptation [6]. 


\section{Social resilience variables}

The three variables in this sub-index included the total Medical Outcomes Study (MOS) Social Support Survey [26], social participation, and a single item measuring perceived loneliness. The total MOS Social Support Survey instrument includes 19 items [26] consisting of the social support elements of emotional/informational support, affection support, tangible support, and positive social interaction. Each question ranges from 1 (none of the time) to 5 (all of the time). The scale has a range of 19-95 with higher scores indicating greater levels of social support. Social participation was a categorical measure developed by researchers at the CLSA. This variable asked the frequency of participation in activities with family or friends in the past 12 months. The answers ranged from "once a day", "at least once a week", "at least once a month", "at least once a year", to "never". This question was recoded into "at least once a week or more" and "at least once a month or less". The social support/participation measures are deemed to be significant resources for adaptation to multimorbidity A single item loneliness ordinal measure assessed how often a participant felt lonely over the past week. This categorical measure ranged from "all of the time, 5-7 days", "occasionally, 3-4 days", "some of the time, 1-2 days" to "rarely or never, less than 1 day". Loneliness is associated with poor multimorbidity outcomes [12].

\section{Psychological resilience variables}

This sub-index included three variables: the Center for Epidemiological Studies Depression (CES-D) Scale [27], the Kessler Psychological Distress K10 Scale [28], and the Diener Satisfaction with Life Scale [29]. The CES-D Scale ranges from 0-28 and contains 10 questions on specific depression symptoms such as hopefulness, appetite and concentration. Each question has possible answers from 0 (rarely or none of the time, less than 1 day) to 3 (most or all of the time, 5-7 days). The Kessler Psychological Distress Scale [28] consists of 10 questions with a total range of $0-30$. Answers to questions can range from 0 (never) to 3 (most of the time). The Depression and distress capture the psychological effects of illness adversity. The Diener Satisfaction with Life Scale [29] ranges from 5 to 35 with higher scores indicating greater life satisfaction. Individual questions range from 1 (strongly disagree) to 7 (strongly agree). It represents positive well-being and adaptation to illness $[12,22]$. Although there is potential overlap of a few items in the depression and distress scales, these were deemed to have minimal effect on the index scores, given the number of items in the scales, and their unique constructs.

In order to standardize different measurement types and skewed distributions of measures, we employed an established and validated mapping system (converting all measures into scores between 0 and 10) using the normalization procedure for creating a frailty index [30], and applied to other areas such as an index of successful aging [31]. As shown in Table 1, ordinal measures were converted by dividing the number of responses into 10 proportionately. Continuous measures (after scale construction) were first converted into quartiles to address skewness, and then scaled to $0,3.3,6.7$ and 10. The three sub-index scores were added and converted back to a range of 0 to 10 (by dividing by 3), and the total composite multimorbidity resilience index was an additive score of the three sub-index scores, and also converted to scores between 0 and 10 (by dividing by 3 ) for comparability. Higher scores indicated greater multimorbidity resilience.

\section{Criterion outcome variables}

Review of the multimorbidity, resilience and aging literature in prior research revealed two primary areas in which we would anticipate associations with multimorbidity resilience. The first of these entails health care utilization. Indeed, extensive research has demonstrated that multimorbidity results in higher health care utilization, especially among older adults [15-17]. We therefore select two measures to assess criterion validity: emergency room visits; and hospital stays. These are expected to reveal inverse associations with the resilience indices.

The second set of variables (pain, perceived health and sleep quality) pertain to aspects of the illness context that affect quality of life, and are anticipated to be associated with multimorbidity resilience. Perception of pain is expected to have an inverse association with resilience, since it has been shown to be a debilitating consequence of multimorbidity $[10,16]$, with direct links to resilience concepts $[2,11]$. Perceived health has been identified as one of the most consistent global measures of health and has been used in a multitude of studies of multimorbidity outcomes $[14,18]$, as well as multimorbidity measurement validation $[1,8]$. Finally, sleep quality has also been associated with multimorbidity outcomes, and represents an important lifestyle factor predicted by deleterious illness experiences [32]. Perceived health and sleep quality are expected to have positive associations with the resilience indices. We use these five variables were used to assess criterion validity.

Health care utilization over the previous year was measured using: a) emergency department visitation, and b) overnight hospital admission coded no (0) and yes (1) in the CLSA dataset. Three ordinal outcome measures associated with illness context were dichotomized, given highly skewed distributions with few cases in some categories: c) pain was dichotomized into none/ 
Table 1 Total Resilience Scale Items, Values and Calculation

\begin{tabular}{|c|c|c|c|c|}
\hline Item & Survey Question & Responses & Value & Score Calculation \\
\hline \multirow[t]{4}{*}{ Summary Performance Score } & & Lowest quartile & 0 & A \\
\hline & & $\begin{array}{l}\text { Second lowest } \\
\text { quartile }\end{array}$ & 3.3 & \\
\hline & & $\begin{array}{l}\text { Second highest } \\
\text { quartile }\end{array}$ & 6.7 & \\
\hline & & Highest quartile & 10 & \\
\hline \multirow[t]{2}{*}{ OARS ADL Scale } & & Lowest quartile & 0 & B \\
\hline & & Remainder & 10 & \\
\hline \multirow[t]{2}{*}{ OARS Instrumental ADL Scale } & & Lowest quartile & 0 & C \\
\hline & & Remainder & 10 & \\
\hline Functional Resilience (FR) & & Derived interval scale & & $(A+B+C) / 3=F R$ \\
\hline \multirow[t]{4}{*}{ Satisfaction with Life Scale } & & Lowest quartile & 0 & $\mathrm{D}$ \\
\hline & & $\begin{array}{l}\text { Second lowest } \\
\text { quartile }\end{array}$ & 3.3 & \\
\hline & & $\begin{array}{l}\text { Second highest } \\
\text { quartile }\end{array}$ & 6.7 & \\
\hline & & Highest quartile & 10 & \\
\hline \multirow{4}{*}{$\begin{array}{l}\text { Center for Epidemiologic Studies Depression } \\
\text { Scale }\end{array}$} & & Highest quartile & 0 & E \\
\hline & & $\begin{array}{l}\text { Second highest } \\
\text { quartile }\end{array}$ & 3.3 & \\
\hline & & $\begin{array}{l}\text { Second Lowest } \\
\text { quartile }\end{array}$ & 6.7 & \\
\hline & & Lowest quartile & 10 & \\
\hline \multirow[t]{4}{*}{ Kessler Psychological Distress Scale } & & Highest quartile & 0 & $\mathrm{~F}$ \\
\hline & & $\begin{array}{l}\text { Second highest } \\
\text { quartile }\end{array}$ & 3.3 & \\
\hline & & $\begin{array}{l}\text { Second lowest } \\
\text { quartile }\end{array}$ & 6.7 & \\
\hline & & Lowest quartile & 10 & \\
\hline Psychological Resilience (PR) & & Derived interval scale & & $(D+E+F) / 3=P R$ \\
\hline \multirow[t]{4}{*}{ MOS Social Support Total Scale } & & Lowest quartile & 0 & G \\
\hline & & $\begin{array}{l}\text { Second lowest } \\
\text { quartile }\end{array}$ & 3.3 & \\
\hline & & $\begin{array}{l}\text { Second highest } \\
\text { quartile }\end{array}$ & 6.7 & \\
\hline & & Highest quartile & 10 & \\
\hline \multirow[t]{4}{*}{ Loneliness } & $\begin{array}{l}\text { How often did you feel lonely in the past } \\
\text { week? }\end{array}$ & $\begin{array}{l}\text { All of the time } \\
\text { (5-7 days) }\end{array}$ & 0 & $\mathrm{H}$ \\
\hline & & $\begin{array}{l}\text { Occasionally } \\
\text { ( } 3-4 \text { days) }\end{array}$ & 3.3 & \\
\hline & & $\begin{array}{l}\text { Some of the time } \\
\text { ( } 1-2 \text { days })\end{array}$ & 6.7 & \\
\hline & & $\begin{array}{l}\text { Rarely or never } \\
(<1 \text { day })\end{array}$ & 10 & \\
\hline \multirow[t]{5}{*}{ Social Participation } & \multirow{5}{*}{$\begin{array}{l}\text { Frequency of participation in family or friends } \\
\text { activities out of the household }\end{array}$} & Never & 0 & । \\
\hline & & At least once a year & 2.5 & \\
\hline & & At least once a month & 5.0 & \\
\hline & & At least once a week & 7.5 & \\
\hline & & At least once a day & 10 & \\
\hline
\end{tabular}


Table 1 Total Resilience Scale Items, Values and Calculation (Continued)

\begin{tabular}{|c|c|c|c|c|}
\hline Item & Survey Question & Responses & Value & Score Calculation \\
\hline Social Resilience (SR) & & Derived interval scale & & $(\mathrm{G}+\mathrm{H}+\mathrm{I}) / 3=\mathrm{SR}$ \\
\hline Total Resilience (TR) & & Derived interval scale & & $\begin{array}{l}(F R+P R+S R) / 3= \\
T R\end{array}$ \\
\hline
\end{tabular}

mild (0) and moderate/severe (1); d) perceived health was based on the single item question "how would you rate your health? This was scored into fair/poor (0) and good/very good/excellent (1); and e) sleep quality was based on the question: how satisfied are you with the quality of your sleep? This variable was coded: very dissatisfied/dissatisfied/neutral (0) and very satisfied/satisfied (1).

\section{Covariates}

Given the importance of several socio-demographic correlates of multimorbidity, we adjusted for the effects of age, gender, education level, total household income, marital status, and region in the logistic regression analyses [8, 13, 31]. In addition, the effects of number of chronic conditions (multimorbidity) was also adjusted in a separate model, given the potential association with resilience. The age variable ranged from 65 to 86 . Gender was a dichotomous variable. The education level variable captured the highest degree, certificate, or diploma obtained by the participant. This variable was dummy coded with the level "no-post-secondary degree, certificate or diploma" as the control, as well as "education below Bachelors: trade certificate or diploma", "Bachelor's degree", to "university degree or certificate above Bachelor's degree" included as well. Total household income was also dummy coded with the level "less than $\$ 20,000$ " as the control; with " $\$ 20,000$ to $\$ 49,999$ ", " $\$ 50,000$ to $\$ 99,999 "$ ", " $\$ 100,000$ to $\$ 149,999 "$ and “\$150,000 or above" as possible responses. The marital status variable was dichotomized from "single, never married or never lived with a partner", "married/living with a partner in a common-law relationship", "widowed", "divorced", and "separated" into "married/common-law" and "non-married/non-common-law" categories. Region was measured based on the five regions typically used in Canadian research: British "Columbia", "Prairies", "Ontario", "Quebec", and "Atlantic" regions. Number of chronic conditions were measured by counting illness categories ( 2 or more) based on 27 available categories.

\section{Data analyses}

Intercorrelations among the resilience indices are presented first. We then employ logistic regression analyses for the dichotomized outcome measures, in order to assess associations with the four resilience indices (e.g. functional, social, psychological and total resilience), adjusting for the socio-demographic variables, as well as number of chronic conditions. The Statistical Package for the Social Sciences, Version 24, was used for the analyses.

\section{Results}

Intercorrelations of resilience indices

All frequencies and descriptive statistics are presented for the resilience measures, outcome variables, and covariates in Table 2. Table 3 shows the intercorrelations of the function, social, and psychological indices and the total resilience composite index, all of which are statistically significant $(p<.001)$. The correlations among the sub-indices ranged between .20 (functional and social domains) and .46 (psychological and social domains), suggesting that they are capturing relatively distinct domains. The correlations between the sub-indices and the total composite index were $.68, .69$ and .82 for the social, functional and psychological domains, respectively. Given that the total index is a composite measure of the sub-indices, it is not surprising that these associations are stronger, with the largest association between the psychological resilience index and the total resilience index. The Cronbach's alpha for the total resilience scale is .69.

\section{Logistic regression analyses of resilience indices and outcome measures}

Table 4 shows the logistic regression results of the three resilience sub-indices and the total resilience measure on the five outcome measures: a), unadjusted; b) controlling for the socio-demographic variables - age, gender, marital status, education, income, and region; and 3) adjusting for the above socio-demographic variables as well as number of chronic conditions. The associations are shown both with and without number of chronic illnesses being adjusted to observe the effects of multimorbidity exceeding two chromic conditions, given the influence that multiple chronic conditions exerts on resilience $[2,14,19]$. In addition, relationships with the criterion outcome variables are statistically significant and in the hypothesized direction, based on the related literature (see Table 4). The associations are most pronounced for the total resilience measure for all five criterion outcome variables, although results for the sub-indices replicate findings for the total index (only results for the total index are discussed below). Confidence 
Table 2 Descriptive Statistics $(n=6771)$

\begin{tabular}{|c|c|c|c|}
\hline Continuous Resilience Index Items & Range & Mean & Standard Deviation \\
\hline Summary Performance Score & 0 to 12 & 7.03 & 2.87 \\
\hline OARS ADL Scale & 4 to 14 & 13.52 & .91 \\
\hline OARS Instrumental ADL Scale & 7 to 14 & 13.84 & .60 \\
\hline Satisfaction with Life Scale & 0 to 35 & 28.16 & 5.85 \\
\hline CES-D Depression Scale & 0 to 28 & 5.41 & 4.51 \\
\hline Kessler Psychological Distress Scale & 10 to 48 & 14.33 & 4.47 \\
\hline MOS Social Support Total Scale & 0 to 64 & 51.15 & 11.02 \\
\hline Ordinal Resilience Index Items & & & Frequency (\%) \\
\hline \multirow[t]{4}{*}{ Loneliness } & \multicolumn{2}{|c|}{ All of the time (5-7 days) } & $164(2.4)$ \\
\hline & \multicolumn{2}{|c|}{ Occasionally (3-4 days) } & $651(9.6)$ \\
\hline & \multicolumn{2}{|c|}{ Some of the time ( $1-2$ days) } & $1000(14.8)$ \\
\hline & \multicolumn{2}{|c|}{ Rarely or never (< 1 day) } & $4956(73.2)$ \\
\hline \multirow[t]{5}{*}{ Social Participation } & \multicolumn{2}{|l|}{ Never } & $55(.8)$ \\
\hline & \multicolumn{2}{|c|}{ At least once a year } & $510(7.5)$ \\
\hline & \multicolumn{2}{|c|}{ At least once a month } & $2558(37.8)$ \\
\hline & \multicolumn{2}{|c|}{ At least once a week } & $3362(49.6)$ \\
\hline & \multicolumn{2}{|c|}{ At least once a day } & $286(4.2)$ \\
\hline Resilience Index Variables & Range & Mean & Standard Deviation \\
\hline Functional Resilience Index & 0 to 10 & 7.09 & 2.61 \\
\hline Psychological Resilience Index & 0 to 10 & 5.19 & 2.92 \\
\hline Social Resilience Index & 0 to 10 & 6.65 & 1.88 \\
\hline Total Resilience Index & .28 to 10 & 6.31 & 1.83 \\
\hline Outcome Variables & & & Frequency (\%) \\
\hline \multirow[t]{2}{*}{ Emergency Department Visitation } & \multicolumn{2}{|l|}{ No } & $5252(77.6)$ \\
\hline & \multicolumn{2}{|l|}{ Yes } & $1519(22.4)$ \\
\hline \multirow[t]{2}{*}{ Hospital Admission - Overnight } & \multicolumn{2}{|l|}{ No } & $5909(87.3)$ \\
\hline & \multicolumn{2}{|l|}{ Yes } & $862(12.7)$ \\
\hline \multirow[t]{2}{*}{ Pain } & \multicolumn{2}{|l|}{ None/Mild } & $4901(72.4)$ \\
\hline & \multicolumn{2}{|c|}{ Moderate/Severe } & $1870(27.6)$ \\
\hline \multirow[t]{2}{*}{ Perceived Health } & \multicolumn{2}{|l|}{ Fair / Poor } & $755(11.2)$ \\
\hline & \multicolumn{2}{|c|}{ Good / Very Good / Excellent } & $6016(88.8)$ \\
\hline \multirow[t]{2}{*}{ Sleep Quality } & \multicolumn{2}{|c|}{ Very Dissatisfied / Dissatisfied / Neutral } & 2599 (38.4) \\
\hline & Very Satisfie & & $4172(61.6)$ \\
\hline Continuous Independent Variables & Range & Mean & Standard Deviation \\
\hline Age & 65 to 86 & 73.16 & 5.68 \\
\hline Number of Chronic Conditions & 2 to 16 & 4.03 & 1.93 \\
\hline Categorical/Ordinal Independent Variables & & & Frequency (\%) \\
\hline Gender & Female & & $3830(56.6)$ \\
\hline & Male & & $2941(43.4)$ \\
\hline Education & No post-se & or diploma & $2081(30.7)$ \\
\hline & Trade certif & & $2101(31.0)$ \\
\hline & Bachelor's & & $1270(18.8)$ \\
\hline & University & bachelor's & $1319(19.5)$ \\
\hline Household Income & Less than $\$$ & & $763(11.2)$ \\
\hline
\end{tabular}


Table 2 Descriptive Statistics ( $n=6771$ ) (Continued)

\begin{tabular}{|c|c|c|}
\hline Continuous Resilience Index Items & Range & Standard Deviation \\
\hline & $\$ 20,000$ to $\$ 49,999$ & $466(6.9)$ \\
\hline & $\$ 50,000$ to $\$ 99,999$ & $2219(32.8)$ \\
\hline & $\$ 100,000$ to $\$ 149,999$ & $2956(43.7)$ \\
\hline & $\$ 150,000$ and over & $367(5.4)$ \\
\hline \multirow[t]{2}{*}{ Marital Status } & Single / Widowed / Divorced / Separated & $2304(34.0)$ \\
\hline & Married / Common-law & $4467(66.0)$ \\
\hline \multirow[t]{5}{*}{ Region } & British Columbia & $1341(19.8)$ \\
\hline & Prairies & $1241(18.3)$ \\
\hline & Ontario & $1532(22.6)$ \\
\hline & Quebec & $1493(22.1)$ \\
\hline & Atlantic & $1163(17.2)$ \\
\hline
\end{tabular}

intervals around the estimated odds ratios (ORs) are very narrow, also indicating small standard errors.

We present three sets of odds ratios (ORs) and 95\% confidence intervals for the total resilience index for the unadjusted and adjusted models. Unadjusted total resilience is associated with: perceived health $(\mathrm{OR}=1.72$, CI $1.65-1.80)$; sleep quality ( $\mathrm{OR}=1.29$, CI $1.26-1.33)$; perceived pain $(\mathrm{OR}=0.74$, CI $0.72-0.76)$; hospital overnight stays $(\mathrm{OR}=0.82$, CI $0.79-0.85)$; and emergency department visits $(\mathrm{OR}=0.86$, CI 0.84-0.89).

After adjusting for the six socio-demographic variables, the associations between the total resilience index and the health outcomes were replicated, with only slight differences in ORs.These include: perceived health $(\mathrm{OR}=1.82$, CI $1.73-1.91)$; sleep quality $(\mathrm{OR}=1.35$, CI 1.31-1.39); perceived pain ( $\mathrm{OR}=0.75$, CI $0.73-0.78)$; hospital overnight stays $(\mathrm{OR}=0.82$, CI $0.79-0.86)$; and emergency department visits $(\mathrm{OR}=0.87$, CI $0.84-0.90)$.

Finally, after also adjusting for number of chronic conditions, relationships were attenuated: perceived health $(\mathrm{OR}=1.68$, CI 1.59-1.77); sleep quality ( $\mathrm{OR}=$ 1.34 , CI 1.30-1.38); perceived pain ( $\mathrm{OR}=0.80$, CI $0.77-$ $0.83)$; hospital overnight stays $(\mathrm{OR}=0.87, \mathrm{CI} 0.83-0.91)$; and emergency department visits ( $\mathrm{OR}=0.90$, CI 0.87 0.94). Results for the sub-indices were also consistent with the hypotheses, but were slightly weaker than for the total resilience measure (see Table 4).

Supplementary analyses were conducted focusing on subsets of three clusters of multimorbid conditions (vascular, osteoporosis, mental health) to decrease the variation of illnesses and symptomologies. The regression analyses replicated the above results (not presented).

\section{Discussion}

This paper provided the rationale and development procedure for a composite index measuring multimorbidity resilience based on three sub-indices (measuring functional, social and psychological domains). Each of the domains were measured using three individual measures of both adversity and resilience, resulting in a continuous measure of total multimorbidity resilience. A standard method is used to standardize the distributions to create a composite index score, based on earlier work on the frailty index [30], and successful aging [31].

Results indicate that the total multimorbidity resilience index is inversely associated with emergency department visits, overnight hospital stats, and perceived pain. In addition, the total index is positively associated with perceived health and sleep quality. These associations are in the hypothesized directions and are replicated after adjusting for socio-demographic factors (age, gender, marital status, education, income and region), as well as number of chronic conditions. In order of strength, the total resilience index is associated with: perceived health; sleep quality; perceived pain; hospital overnight stays; and emergency department visits. These criterion variables represent important outcomes of multimorbidity experience among older adults with important health and health care implications.

Table 3 Inter-Correlation Matrix for Multimorbidity Resilience Indexes, Weighted $(n=6771)$

\begin{tabular}{lllll}
\hline & Functional Resilience & Psychological Resilience & Social Resilience & Total Resilience \\
\hline Functional Resilience & - & - & - & $.69^{* * *}$ \\
Psychological Resilience & $.28^{* * *}$ & - & - & $.82^{* * *}$ \\
Social Resilience & $.20^{* * *}$ & $.46^{* * *}$ & - & $.68^{* * *}$ \\
\hline
\end{tabular}

*** $p<.001$ 


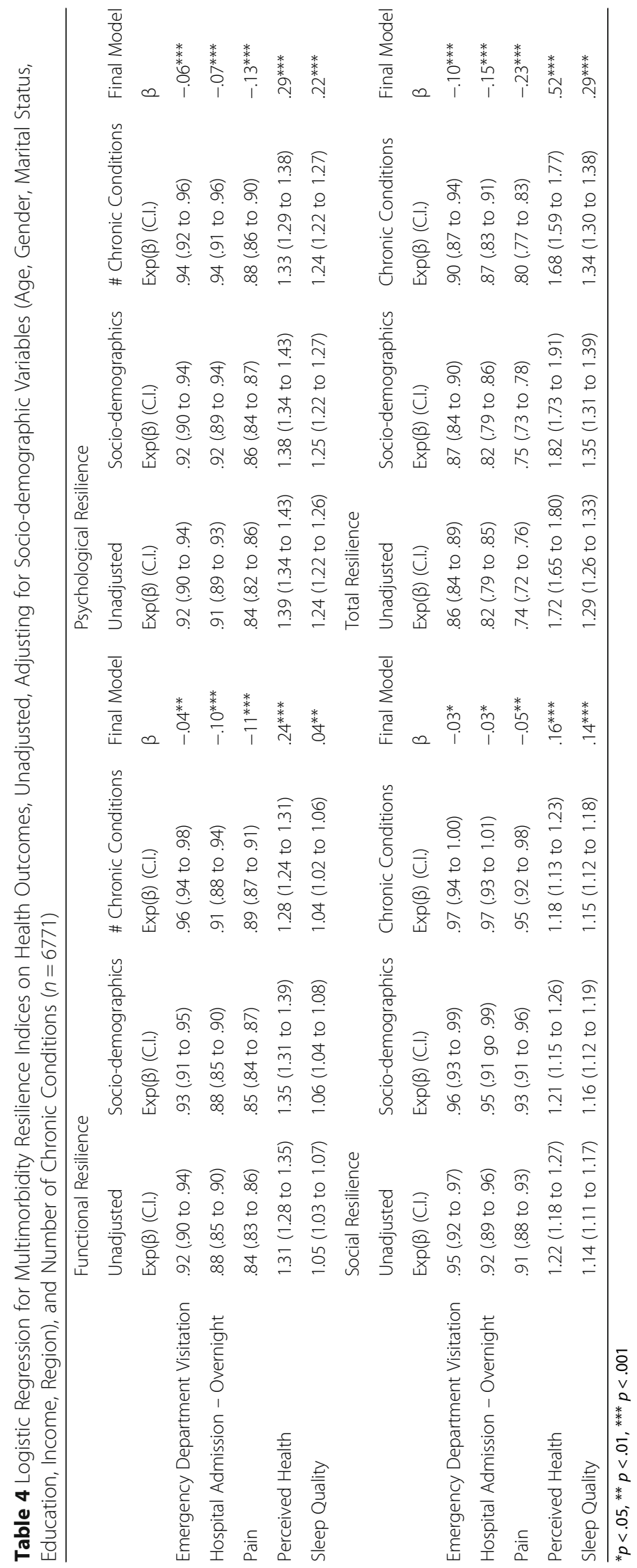


Comparisons of the criterion validity analyses using our composite resilience index and other resilience measures provides additional evidence of criterion-related validity. Although the available literature focusing on chronic illness, especially among older adults is sparse, there are some useful comparative studies. The Brief Resilient Coping Scale (BRCS), a 4-item measure of tendencies to cope with stress in an adaptive manner, has been shown to have adequate internal consistency (baseline Cronbach's alpha $=.64$ ) and test-retest reliability (.71) [21]. Using two samples of persons (mean ages 46 and 58, respectively) with rheumatoid arthritis, the BRCS did not correlate with age, employment stats, length of time since diagnosis, pain, or fatigue in either sample at baseline [21]. However, predictable correlations were found with pain coping behaviours and psychological well-being, the former of which is consistent with the multimorbidity resilience index.

In a study of general resilience (measured by cumulative lifetime adversity, social support and mastery) over a two-year period based on persons aged 50-70 drawn from the US Health and Retirement Survey, researchers supported an inverse association with hospital utilization $\mathrm{OR}=0.75$, CI 0.64-0.86), and a positive association with self-rated health (OR $=1.49$, CI 1.17-1.88), after adjusting for socio-demographic and lifestyle covariates [32]. These associations are virtually identical (but slightly weaker), to the ones found in our CLSA study using the composite multimorbidity resilience index for overnight hospital admissions ( $\mathrm{OR}=.87, \mathrm{CI} .83-.91)$, and perceived health $(\mathrm{OR}=1.68$, CI 1.59-1.77), after adjusting for all covariates.

Another study of general resilience (measured as a stressful event within 5 years, level of stressfulness and level of recovery), analyzed a sample of 546 non-disabled older adults, i.e. who did not require personal assistance, aged $70+$ living in the US [33]. While non-disabled, 56\% of their sample had two or more chronic conditions, making them comparable to the CLSA sample. The researchers found associations between their six-item resilience measure and functional status, depression and self-rated health (SRH). Of particular relevance, their findings for SRH were consistent with ours $(\mathrm{OR}=1.38$, CI 1.01-1.79) after adjusting for socio-demographic and functional measures. In addition, other studies have shown support for associations between resilience and pain, as well as sleep, although not directly comparable to the CLSA sample [2, 34]. Taken together, review of available studies show that our results of the criterion validity outcome analyses using the total multimorbidity resilience index are comparable.

There are a number of limitations of this research. First, cross-sectional study designs can not capture change in resilience levels and outcomes, which is needed to substantiate this work. Further, we are unable to draw conclusions regarding the direction of causality due to the temporal nature of these data. As more waves of the CLSA become available, the resilience indices can be validated with longitudinal data. Second, there are other methods of standardizing the resilience sub-indices and total index scores with respect to weighting and item inclusion. The current method is informed by the procedure used in the development of the Frailty Index [30] and Successful Aging Index [31]. Third, some level of granularity is lost when converting continuous scores into quartiles. Fourth, some of the items in the composite resilience index overlap (especially, the Kessler Psychological Distress K10 scale, the CES-D scale, and the loneliness item). The large number of items and scoring system mitigates the extent to which this biases the results.

\section{Conclusions}

This study developed a new multimorbidity resilience index comprised of functional, social and psychological domains with measures of adversity and adaptation. The criterion validation of the index and comparisons with similar studies provides initial support for this new measure. Although the study does not account for the stage of the comorbidity and its severity, the consistency of associations with hypothesized outcomes has several implications. First, a composite resilience index can be used to identify individuals at higher risk of emergency department visits and/or hospitalization, as well as perceptions of health, pain and sleep quality. Second, a composite resilience measure can be extended to other areas of risk, such as injury and falls. Third, identification of individuals at lower levels of resilience can be helpful in interventions aimed at improving independent community living. All of these clinical implications can potentially lower health care costs and extend longevity.

Further confirmatory research is needed to validate the resilience indices using other known data sets, such as the US Health and Retirement Study. In addition, these measures need to be incorporated into explanatory and predictive models in order to identify and compare determinates and outcomes, especially using longitudinal data sources. Research is also warranted to establish the full usefulness of this measure among different populations (e.g., race, ethnicity, socio-economic status, etc.), as well as applications to relevant clinical settings.

\section{Additional file}

Additional file 1: Universities and organizations participating in the harmonized CLSA ethics. (DOCX 13 kb)

\section{Abbreviations}

ADL: Activities of Daily Living; CCHS: Canadian Community Health Survey; CED-D: Center for Epidemiological Studies Depression; CIHR: Canadian Institutes of Health Research; CLSA: Canadian Longitudinal Study of Aging; 
HRQL: Health related quality of life; IADL: Instrumental activities of daily living LMMR - Lifecourse model of multimorbidity resilience; MOS: Medica Outcomes Study; OARS: Older Americans Resources and Services; SFU ORS: Simon Fraser University, Office of Research Services

\section{Acknowledgements}

This research was made possible using the data/biospecimens collected by the Canadian Longitudinal Study on Aging (CLSA). The opinions expressed in this manuscript are the authors; own and do not reflect the views of the CLSA.

\section{Funding}

Funding for the Canadian Longitudinal Study on Aging (CLSA) data collection is provided by the Government of Canada through the Canadian Institutes of Health Research (CIHR) under grant reference: LSA 9447 and the Canada Foundation for Innovation. This research has been conducted using the CLSA Comprehensive Cohort dataset [version 1.0], under Application Number [\#150914] (https://www.clsa-elcv.ca/). The current study was funded through a CIHR CLSA Catalyst Grant (RN302177-373073). TC is funded by a CIHR Postdoctoral Fellowship (MFE146676).

\section{Availability of data and materials}

The CLSA data can be publicly obtained through the CLSA Data Application Process (https://www.clsa-elcv.ca/).

\section{Authors' contributions}

AW - Main writer of manuscript; SL, NS, BM, DM, TC - Secondary authors made substantial contributions to the conception and design of the manuscript, and/or interpretation of the data, and all participated in editing and revising the manuscript; IF - Conducted analyses of data, drafting of the methods section, and revisions. All authors have read and approved the final version of the manuscript, and have agreed to be accountable for all parts of the manuscript.

\section{Ethics approval and consent to participate}

This current project received ethics approval at two levels. Consent to participate was obtained for all participants under the CLSA harmonized multi-university ethics process that was centrally organized and approved by the Hamilton Integrated Research Ethics Board (HiREB), Hamilton Health Sciences/McMaster University. Written consent was obtained from all CLSA participants prior to enrollment in the CLSA study via a fully disclosed consent form approved by the ethics committees of all 11 Canadian participating universities (see Additional file 1). Individuals who were not deemed to be cognitively functional were excluded from the CLSA study. Simon Fraser University (SFU) was a participating institution in the CLSA data collection, and the SFU Office of Research Services Ethics Committee reviewed all consent material prior to data collection. Harmonized ethics for the data collection was approved by the Simon Fraser University, Office of Research Services (SFU ORS \#2010 s0281). For the current study, ethics approval was obtained from the Office of Research Services (ORS) Ethics Committee at Simon Fraser University as a Minimal Risk Project (\#2016 s0281). The requirement for further written informed consent was waived by the SFU ORS Ethics Committee.

\section{Consent for publication}

Not applicable.

\section{Competing interests}

The authors declare that they have no competing interests.

\section{Publisher's Note}

Springer Nature remains neutral with regard to jurisdictional claims in published maps and institutional affiliations.

\section{Author details}

'Department of Gerontology, and Gerontology Research Centre, Simon Fraser University, 2800-515 Hastings Street, Vancouver, BC V6B 5K3, Canada. ${ }^{2}$ Faculty of Health Sciences, Simon Fraser University, 8888 University Drive, Burnaby, BC V5A 1S6, Canada. 3Department of Geography, Simon Fraser University, 8888 University Drive, Burnaby, BC V5A 156, Canada. ${ }^{4}$ Department of Biomedical Physiology \& Kinesiology, 8888 University Drive, Burnaby, BC
V5A 1S6, Canada. ${ }^{5}$ Departments of Gerontology and Sociology/ Anthropology, Simon Fraser University, 2800-515 Hastings Street, Vancouver, BC V6B 5K3, Canada. 'Gerontology Research Centre, Simon Fraser University, 2800-515 Hastings Street, Vancouver, BC V6B 5K3, Canada. ${ }^{7}$ Gerontology Research Centre, Simon Fraser University, 2800-515 West Hastings St. Vancouver V6B 5K3, Canada. ${ }^{8}$ Department of Gerontology, Simon Fraser University, 2800-515 Hastings Street, Vancouver, BC V6B 5K3, Canada.

Received: 10 January 2018 Accepted: 27 June 2018

Published online: 27 July 2018

\section{References}

1. Resnick B, Galk E, Dorsey S, Scheve A, Gutkin S. Reliability and validity testing of the physical resilience measure. The Gerontologist. 2011:51(5): 643-52. https://doi.org/10.1093/geront/gnr016.

2. Wiles J, Wild K, Kerse N, Allen R. Resilience from the point of view of older people: There's still life beyond the funny knee'. Soc Sci Med. 2012;74:416-24.

3. Windle G. What is resilience? A review and concept analysis. Rev Clin Gerontol. 2011;21(2):152-69. https://doi.org/10.1017/S0959259810000420.

4. Connor K, Davidson J. Development of a new resilience scale: the ConnorDavidson resilience scale (CD-RISC). Depress Anxiety. 2003;18:76-82.

5. Cosco T, Kaushal A, Hardy R, Richards M, Kuh D, Stafford M. Operationalising resilience in longitudinal studies: a systematic review of methodological approaches. J Epidemiol Community Health. 2017:77(1):98-104.

6. Silverman A, Molton I, Alschuler K, Ehde D, Jensen M. Resilience predicts functional outcomes in people aging with disabilities: a longitudinal investigation. Arch Phys Med Rehabil. 2015;96:1262-8.

7. Windle G, Bennett K, Noyes J. A methodological review of resilience measurement scales. Health Qual Life Outcomes. 2011;9:8. https://doi.org/ 10.1186/1477-7525-9-8.

8. Wister A, Levasseur M, Griffith L, Fyffe I. Estimating multiple morbidity disease burden among older persons: a convergent construct validity study to discriminate between six chronic illness measures, CCHS 2008/09. Biomedical Central Geriatrics. 2015;15(12) https://doi.org/10.1186/s12877015-0001-8.

9. Sells D, Sledge WH, Wieland M, Walden D, Flanagan E, Miller R, Davidson L. Cascading crises, resilience, and social support within the onset and development of multiple chronic conditions. Chronic Illness. 2009;5(2):92102. https://doi.org/10.1177/1742395309104166.

10. Trivedi $R$, Bosworth $H$, Jackson G. Resilience in chronic illness. In: Resnick B, Gwyther L, Roberto K, editors. Resilience in aging: concepts, research and outcomes. New York, NY: Springer; 2011. p. 45-63.

11. Stewart D, Yuen T. A systematic review of resilience in the physically ill. Psychosomatics. 2011;52(3):199-209.

12. Wister, A., Coatta, K., Schuurman, N., Lear, S., Rosin, M. \& MacKey, D. (2016).A Lifecourse model of resilience applied to aging with multimorbidity. International Journal of Aging \& Human Development. doi: https://doi.org/ 10.1177/0091415016641686.

13. Salive M. Multimorbidity in older adults. Epidemiol Rev. 2013;35:75-83. https://doi.org/10.1093/epirev/mxs009.

14. Wister A, Kendig H, Mitchell BA, Fyffe I, Loh V. Multimorbidity, health and aging in Canada and Australia: a tale of two countries. Biomedical Central Geriatrics. 2016;16(1) https://doi.org/10.1186/s12877-016-0341-z.

15. Agborsangaya CB, Lau D, Lahtinen M, Cooke T, Johnson J. Health-related quality of life and healthcare utilization in multimorbidity: results of a crosssectional survey. Qual Life Res. 2013;22:791-9. https://doi.org/10.1007/ s11136-012-0214-7.

16. Institute of Medicine. Living well with chronic illness: a call for public health action. Washington, DC: The National Academies Press; 2012

17. Tinetti ME, MCAvay GJ, Chang SS, Newman AB, Fitzpatrick AL, Fried TR, Peduzzi PN. Contributions of multiple chronic conditions to universal health outcomes. J Am Geriatr Soc. 2011;59(9):1686-91. https://doi.org/10.1111/j. 1532-5415.2011.03573.x

18. Galenkamp H, Braam A, Huisman M, Deeg D. Somatic multimorbidity and self-rated health in the older population. J Gerontol. 2011;66B(3):380-6. https://doi.org/10.1093/geronb/gbr032.

19. Rybarczyk B, Emery E, Guequierre L, Shamaskin A, Behel J. The role of resilience in chronic illness and disability in older adults. In: Hayslip B, Smith G, editors. Annual review of gerontology and geriatrics, volume 32, 2012, special issue: emerging perspectives on resilience in adulthood and later life. New York: Springer; 2012. p. 173-88. 
20. Connelly E, Allen C, Hatfield K, Palma-Oliveira J, Woods D, Linkov I. Features of resilience. Environ Syst Decisions. 2017;37(1):46-50

21. Sinclair $\mathrm{V}$, Wallston $\mathrm{K}$. The development and psychometric evaluation of the brief resilient coping scale. Assessment. 2004;11(1):94-101.

22. Pearlin LI, Schieman S, Fazio EM, Meersman S. Stress, health, and the life course: some conceptual perspectives. J Health Soc Behav. 2005;46(2):205-19. https://doi.org/10.1177/002214650504600206.

23. Raina P, Wolfson C, Kirkland S, Griffith L, Oremus M, Wister AV, et al. The Canadian longitudinal study of aging. Can J Aging. 2009;28(3):221-9.

24. Fillenbaum GG, Smyer MA. The development, validity, and reliability of the OARS multidimensional functional assessment questionnaire. J Gerontol. 1981;36(4):428-34. https://doi.org/10.1093/geronj/36.4.428.

25. Guralnik JM, Simonsick EM, Ferrucci L, Glynn RJ, Berkman LF, Blazer DG, Scherr PA, Wallace RB. A short physical performance battery assessing lower extremity function: association with self-reported disability and prediction of mortality and nursing home admission. J Gerontol. 1994;49(2):85-94 https://doi.org/10.1093/geronj/49.2.M85.

26. Sherbourne CD, Stewart AL. The MOS social support survey. Soc Sci Med. 1991;32(6):705-14. https://doi.org/10.1016/0277-9536(91)90150-B.

27. Radloff LS. The CES-D scale: a self-report depression scale for research in the general population. Appl Psychol Meas. 1977;1(3):385-401. https://doi.org/ 10.1177/014662167700100306.

28. Kessler RC, Andrews G, Colpe LJ, Hiripi E, Mroczek DK, Normand S-LT, Walters EE, Zaslavsky AM. Short screening scales to monitor population differences and trends in non-specific psychological distress. Psychol Med. 2002:32:959-76. https://doi.org/10.1017/S0033291702006074.

29. Diener E, Emmons RA, Larsen RJ, Griffin S. The satisfaction with life scale. J Pers Assess. 1985:49(1):71-5. https://doi.org/10.1207/s15327752jpa4901_13.

30. Searle S, Mitnitski A, Gahbauer E, Gill T, Rockwood K. A standard procedure for creating a frailty index. Biomedical Central Geriatrics. 2008;8(24) https://doi.org/10.1186/1471-2318-8-24.

31. Cosco T, Stephan B, Brayne C. Validation of an a priori, index model of successful aging in a population-based cohort study: the successful aging index. Int Psychogeriatr. 2015;27(12):1971-7. https://doi.org/10.1017/ S1041610215000708

32. Ezeamama A, Elkins J, Simpson C, Smith S, Allegra J, Miles T. Indicators of resilience and healthcare outcomes: findings from the 2010 health and retirement survey. Qual Life Res. 2016;25:1007-15. https://doi.org/10.1007/ s11136-015-1144-y.

33. Hardy S, Concato J, Gill T. Resilience of community-dwelling older persons. J Am Geriatr Soc. 2004;52:257-62.

34. Segovia L, Moore J, Lhnville S, Hoyt R, Hain R. Sleep and resilience: a longitudinal 37-year follow-up study of Vietnam repatriated prisoners of war. Mil Med. 2013;78(2):196.

\section{Ready to submit your research? Choose BMC and benefit from:}

- fast, convenient online submission

- thorough peer review by experienced researchers in your field

- rapid publication on acceptance

- support for research data, including large and complex data types

- gold Open Access which fosters wider collaboration and increased citations - maximum visibility for your research: over $100 \mathrm{M}$ website views per year 